\title{
High-purity pulsed squeezing generation with integrated photonics
}

\author{
Chaohan Cui, Christos N. Gagatsos $\mathbb{C}^{0}$, Saikat Guha, and Linran Fan ${ }^{*}{ }^{*}$ \\ James C. Wyant College of Optical Sciences, The University of Arizona, Tucson, Arizona 85721, USA
}

(Received 30 July 2020; revised 5 August 2020; accepted 12 January 2021; published 26 February 2021)

\begin{abstract}
Squeezed light has evolved into a powerful tool for quantum technology, ranging from quantum-enhanced sensing and quantum-state engineering based on partial postselection techniques. The pulsed generation of squeezed light is of particular interest, as it can provide accurate time stamps and a physically defined temporal mode, which are highly preferred in complex communication networks and large-scale information processing. However, the multimode feature of pulsed squeezing limits the purity of the output state, negatively impacting its application in quantum technology. Previous demonstrations and analysis of pulsed squeezing focus on single-pass configurations and synchronously pumped free-space cavities. In this paper, we propose a different approach to generate pulsed squeezing with high temporal purity, where a parametric down-conversion process in integrated photonic cavities is pumped by a single-pass pulse. We show that the effective mode number of the output pulsed squeezing approaches unity. Such a high-purity squeezed light can be realized with broad parameters and a low pump power, providing a robust approach to generating large-scale quantum resources.
\end{abstract}

DOI: 10.1103/PhysRevResearch.3.013199

\section{INTRODUCTION}

Non-Gaussian states are indispensable resources required by quantum information processing to demonstrate quantum advantage [1]. Partial detection of squeezed light is one of the most important optical approaches to generate non-Gaussian states [2-6]. Optical cat and kitten states have been generated based on photon-number subtraction from a single-mode squeezed vacuum [7-10]. In principle, arbitrary non-Gaussian states, including the Gottesman-Kitaev-Preskill (GKP) state for cluster-modal quantum computing [11-17], can be generated based on the Gaussian-boson-sampling (GBS) configuration and the photon-number-resolving (PNR) detection $[18,19]$. One critical requirement to implement partial detection of squeezed light is that all photons must be in the same spectral-temporal mode. Otherwise, unconditioned Gaussian modes will be mixed with the target non-Gaussian mode, thus decreasing the purity of the output state. Common techniques utilized for single photons, such as spectral filtering and postselection within a small time window, cannot be applied for the squeezed light due to the excessive loss. Improved pulsed squeezing also finds applications beyond engineering of non-Gaussian states of light. For example, imaging of delicate biological samples can benefit from pulsed squeezing by improving the sensitivity beyond the standard quantum limit [20].

\footnotetext{
*Ifan@optics.arizona.edu

Published by the American Physical Society under the terms of the Creative Commons Attribution 4.0 International license. Further distribution of this work must maintain attribution to the author(s) and the published article's title, journal citation, and DOI.
}

One standard configuration to implement pulsed squeezing is the single-pass parametric down-conversion [21-25]. This process intrinsically involves multiple modes in both space and time, which all have significant squeezing and energy [26]. Synchronously pumped parametric down-conversion in free-space cavities has also been proposed for pulsed squeezing [27,28]. However, the generated squeezing still contains significant multimode contribution. It also requires that the pump repetition rate matches the cavity free-spectra range (FSR), which is challenging for integrated cavities with a large FSR. While the complex shaping of local oscillators can be utilized to improve the measured squeezing level [29], it does not work well on non-Gaussian state generation through partial detection, which requires the separation of different modes. Therefore, a different approach to generating pulsed squeezing with high purity is highly desired to further improve the capability of photonic quantum information processing.

In this paper, we analyze pulsed squeezing with an unexplored configuration: the cavity-enhanced parametric down-conversion with a single-pass pulsed pump. Such configuration is well positioned for pulsed squeezing generation with integrated photonic cavities, with the FSR much larger than the bandwidth of the pump pulse. The Bloch-Messiah approach is used to decompose the input-output relation into independent squeezing modes [30,31]. We demonstrate that the effective mode number at the output can approach unity, showing there is only one dominant spectral-temporal mode. Unlike the single-pass pulsed squeezing [26], this approach does not require the delicate shaping of the pump, making its experimental implementation robust.

\section{THEORETICAL MODEL}

The proposed configuration is shown in Fig. 1 and consists of a photonic ring cavity evanescently coupled to a bus 


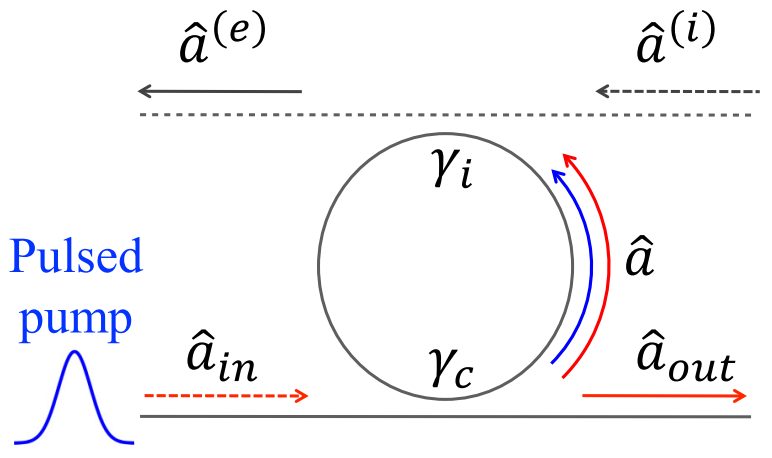

FIG. 1. Proposed configuration for high-purity pulsed squeezing generation: parametric down-conversion in a photonic ring cavity with a pulsed pump. The solid line represents the bus waveguide with signal coupling rate $\gamma_{c}$; the dotted line represents the virtual waveguide for the intrinsic loss channel with loss rate $\gamma_{i}$. The dotted arrows indicate the input vacuum $\hat{a}_{\text {in }}$ and $\hat{a}^{(i)}$; the solid arrows indicate the output mode $\hat{a}_{\text {out }}$ and $\hat{a}^{(e)}$.

waveguide. Phase-matching condition is satisfied for the degenerate parametric down-conversion between the pump mode centering at frequency $2 \omega_{0}$ and the signal mode centering at frequency $\omega_{0}$. The input pump pulse with a center frequency $2 \omega_{0}$ is launched into the cavity through the bus waveguide.

The dynamics of the intracavity signal mode $\hat{a}$ can be described by the equation of motion $[32,33]$

$$
\frac{d \hat{a}}{d t}=\frac{i}{\hbar}[\hat{H}, \hat{a}]-\frac{\gamma}{2} \hat{a}+\sqrt{\gamma_{i}} \hat{a}^{(i)}+\sqrt{\gamma_{c}} \hat{a}_{\text {in }}
$$

with intrinsic loss rate $\gamma_{i}$, bus waveguide coupling rate $\gamma_{c}$, total cavity decay $\gamma=\gamma_{i}+\gamma_{c}$, and noise operators due to intrinsic loss $\hat{a}^{(i)}$ and bus waveguide $\hat{a}_{\text {in }}$. The Hamiltonian $\hat{H}$ can be written as

$$
\begin{aligned}
\hat{H}= & \hbar \omega_{0} \int d \omega \hat{a}^{\dagger}(\omega) \hat{a}(\omega) \\
& +\frac{i \hbar \kappa}{2} \iint d \omega d \omega^{\prime} \hat{a}^{\dagger}(\omega) \hat{a}^{\dagger}\left(\omega^{\prime}\right) \varepsilon\left(\omega+\omega^{\prime}\right)+\text { H.c. }
\end{aligned}
$$

with $\kappa$ the single-photon coupling rate for parametric down-conversion. The intracavity pump field $\varepsilon(\omega)$ is written as

$$
\varepsilon(\omega)=E_{p}(\omega) \frac{\sqrt{\gamma_{p c}}}{-i\left(\omega-2 \omega_{0}\right)+\gamma_{p} / 2}
$$

with the bus waveguide coupling rate $\gamma_{p c}$ and total decay rate $\gamma_{p}$ for the pump mode, and the spectrum amplitude of the input pulse $E_{p}(\omega)$. Utilizing Fourier transformation, Eq. (1) can be further expressed in the frequency domain

$$
\begin{aligned}
0= & \int d \omega^{\prime}\left[i\left(\omega-\omega_{0}\right)-\frac{\gamma}{2}\right] \delta\left(\omega-\omega^{\prime}\right) \hat{a}\left(\omega^{\prime}\right) \\
& +\int d \omega^{\prime} \kappa \varepsilon\left(\omega+\omega^{\prime}\right) \hat{a}^{\dagger}\left(\omega^{\prime}\right) \\
& +\sqrt{\gamma_{i}} \hat{a}^{(i)}(\omega)+\sqrt{\gamma_{c}} \hat{a}_{\text {in }}(\omega) .
\end{aligned}
$$

By including the complex conjugation, we can rewrite Eq. (4) into the following equivalent matrix form

$$
\begin{aligned}
0= & \left(\begin{array}{cc}
D & E \\
E^{\dagger} & D^{\dagger}
\end{array}\right)\left(\begin{array}{c}
\hat{a}(\omega) \\
\hat{a}^{\dagger}(\omega)
\end{array}\right) \\
& +\sqrt{\gamma_{i}}\left(\begin{array}{c}
\hat{a}^{(i)}(\omega) \\
\hat{a}^{(i) \dagger}(\omega)
\end{array}\right)+\sqrt{\gamma_{c}}\left(\begin{array}{c}
\hat{a}_{\mathrm{in}}(\omega) \\
\hat{a}_{\mathrm{in}}^{\dagger}(\omega)
\end{array}\right),
\end{aligned}
$$

where the diagonal matrix $D\left(\omega, \omega^{\prime}\right)=\left[i\left(\omega-\omega_{0}\right)-\right.$ $\gamma / 2] \delta\left(\omega-\omega^{\prime}\right)$ shows the effect of frequency detuning and energy damping, and the matrix $E\left(\omega, \omega^{\prime}\right)=\kappa \varepsilon\left(\omega+\omega^{\prime}\right)$ shows the nonlinear interaction enhanced by the pump. The output field can then be derived based on the input-output theory

$$
\begin{aligned}
\left(\begin{array}{c}
\hat{a}_{\text {out }}(\omega) \\
\hat{a}_{\text {out }}^{\dagger}(\omega)
\end{array}\right)= & {\left[\left(\begin{array}{ll}
I & \\
& I
\end{array}\right)+\gamma_{c}\left(\begin{array}{cc}
D & E \\
E^{\dagger} & D^{\dagger}
\end{array}\right)^{-1}\right]\left(\begin{array}{l}
\hat{a}_{\text {in }}(\omega) \\
\hat{a}_{\text {in }}^{\dagger}(\omega)
\end{array}\right) } \\
& +\sqrt{\gamma_{c} \gamma_{i}}\left(\begin{array}{cc}
D & E \\
E^{\dagger} & D^{\dagger}
\end{array}\right)^{-1}\left(\begin{array}{c}
\hat{a}^{(i)}(\omega) \\
\hat{a}^{(i) \dagger}(\omega)
\end{array}\right) .
\end{aligned}
$$

Through the derivation, we assume that the intrinsic loss of the photonic cavity is Markovian and can be modeled as a virtual waveguide with the input mode $\hat{a}^{(i)}$ and output mode $\hat{a}^{(e)}$ (dashed line in Fig. 1) [33,34]. Then Eq. (6) is converted to the symplectic form

$$
\begin{aligned}
& \left.\left(\begin{array}{c}
\hat{a}_{\text {out }}(\omega) \\
\hat{a}_{\text {out }}^{\dagger}(\omega) \\
\hat{a}^{(e)}(\omega) \\
\hat{a}^{(e) \dagger}(\omega)
\end{array}\right)=\left(\begin{array}{cc}
{\left[\begin{array}{cc}
I & \\
& I
\end{array}\right)+\gamma_{c}\left(\begin{array}{cc}
D & E \\
E^{\dagger} & D^{\dagger}
\end{array}\right)^{-1}} & \begin{array}{c}
\sqrt{\gamma_{c} \gamma_{i}}\left(\begin{array}{cc}
D & E \\
E^{\dagger} & D^{\dagger}
\end{array}\right)^{-1} \\
\sqrt{\gamma_{c} \gamma_{i}}\left(\begin{array}{cc}
D & E \\
E^{\dagger} & D^{\dagger}
\end{array}\right)^{-1}
\end{array} \quad\left[\begin{array}{cc}
I & \\
& I
\end{array}\right)+\gamma_{i}\left(\begin{array}{cc}
D & E \\
E^{\dagger} & D^{\dagger}
\end{array}\right)^{-1}
\end{array}\right]\right)\left(\begin{array}{c}
\hat{a}_{\text {in }}(\omega) \\
\hat{a}_{\text {in }}^{\dagger}(\omega) \\
\hat{a}^{(i)}(\omega) \\
\hat{a}^{(i) \dagger}(\omega)
\end{array}\right)
\end{aligned}
$$

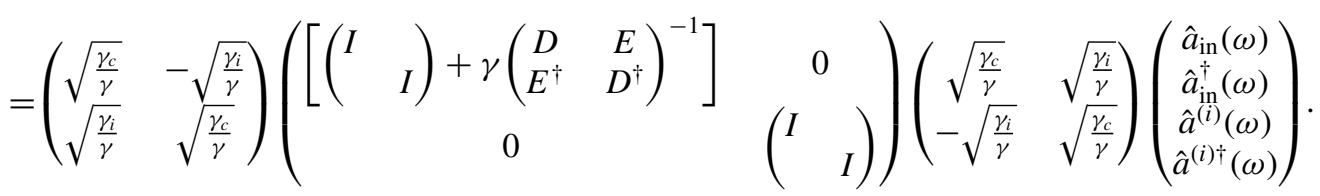

The Bloch-Messiah decomposition means that an arbitrary Gaussian transformation can be represented by two passive linearoptical interferometers ( $P$ and $Q$ in our case) with parallel squeezing transformations. This allows us to rewrite the core matrix $\left[\begin{array}{ll}{ }^{I} & I\end{array}\right)+\gamma\left(\begin{array}{cc}D & E \\ E^{\dagger} & D^{\dagger}\end{array}\right)$ ] $]$ into a combination of a set of single-mode squeezers (Bogoliubov transformations) placed between two 
multiport interferometers [30,31]:

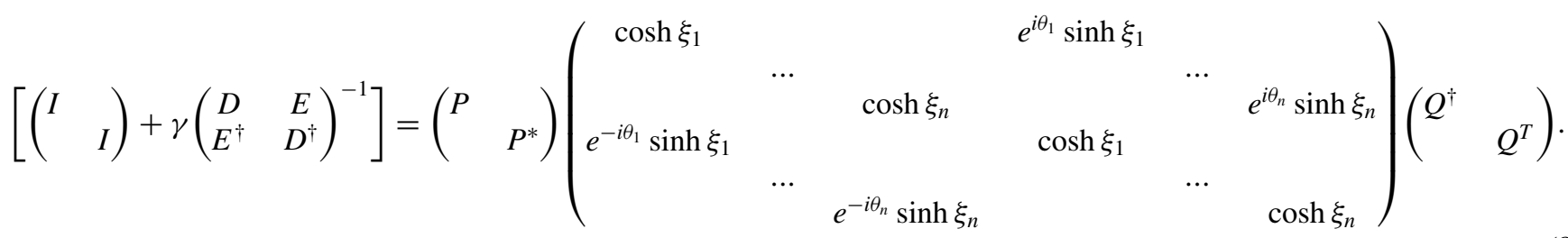

Therefore, the overall input-output relation is modeled as a multimode optical parametric amplifier sandwiched by two beam splitters with reflectivity $R=\gamma_{c} / \gamma$ (Fig. 2). The spectral-temporal shape of the characteristic input mode of each single-mode squeezer $\left(b_{i n, k}\right)$ is determined by the unitary transformation $Q$ :

$$
\hat{b}_{\text {in }}=Q^{\dagger}\left(\sqrt{\frac{\gamma_{c}}{\gamma}} \hat{a}_{\text {in }}(\omega)+\sqrt{\frac{\gamma_{i}}{\gamma}} \hat{a}^{(i)}(\omega)\right) .
$$

Each characteristic mode undergoes independent squeezing

$$
\hat{b}_{\text {out }, k}=\cosh \xi_{k} \hat{b}_{i n, k}+e^{i \theta_{k}} \sinh \xi_{k} \hat{b}_{i n, k}^{\dagger},
$$

where $\xi_{k}$ and $\theta_{k}$ are the squeezing amplitude and phase of the $k$ th mode. After mixing with vacuum at the second beam splitter, the variance of the squeezed quadrature of the $k$ th mode is

$$
\left\langle\left[\Delta X_{k}\left(\frac{\theta_{k}-\pi}{2}\right)\right]^{2}\right\rangle=\frac{1}{2}\left(\frac{\gamma_{i}}{\gamma}+\frac{\gamma_{c}}{\gamma} e^{-2 \xi_{k}}\right) .
$$

This result is identical to the continuous-wave-squeezing (CW-squeezing) process [32], where the maximum squeezing is limited by the intrinsic loss of the cavity. Overcoupled cavity $\left(\gamma_{c} \gg \gamma_{i}\right)$ is required to realize high squeezing. The effective mode number $K$ can be directly calculated from the squeezing amplitude $\xi_{k}$ :

$$
K=\frac{\left(\sum_{k} \sinh ^{2} \xi_{k}\right)^{2}}{\sum_{k} \sinh ^{4} \xi_{k}} .
$$

Higher mode number $K$ implies a lower purity [35]. Since the squeezed light includes multiphoton components, the definition of purity is more general than the one for the singlephoton case, where only the first-order term is considered.

Our derivation assumes that the pump is below threshold so that all the output modes are still squeezed vacuum. The pump threshold can be determined when the gain for any intracavity mode is equal to the amplitude loss rate $\gamma / 2$. By

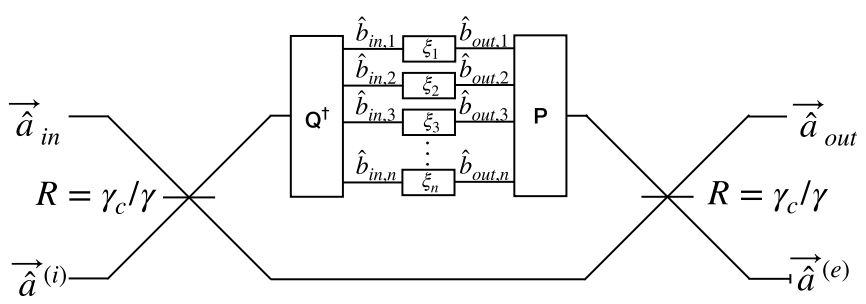

FIG. 2. Equivalent photonic circuit of parametric downconversion in a photonic cavity with pulsed pump. rewriting Eq. (1) into the rotation frame $\hat{a} \rightarrow \hat{a} e^{i \omega_{0} t}$ and taking the average on the initial vacuum state, we can express the classical dynamics of the intracavity field as

$$
\langle\dot{\hat{a}}\rangle=-\frac{\gamma}{2}\langle\hat{a}\rangle+E\langle\hat{a}\rangle^{*} .
$$

The solution to Eq. (13) has the form $\langle\dot{\hat{a}}\rangle_{k}=S_{k} e^{\lambda_{k} t}$, where $\lambda_{k}$ and $S_{k}$ are the $k$ th eigenvalue and eigenstate of matrix $E$ [27]. We label the eigenvalue with the largest modulus as $\lambda_{0}$. As $\lambda_{0}$ can always be made a real number by adjusting the global phase, the criteria for the threshold pump becomes $\lambda_{0}=\gamma / 2$. It is noteworthy that the eigenstates of the intracavity modes (decomposition of matrix $E$ ) are different from the characteristic modes obtained at the output with the Bloch-Messiah decomposition of the core matrix in Eq. (7).

\section{NUMERICAL SIMULATION}

As can be seen from the last section, all critical properties of the output state depend on the distribution of squeezing amplitude $\xi_{k}$. In order to get further insight, the generation of pulsed squeezing in photonic ring cavities is investigated numerically. Without loss of generality, we assume the input pump has a Gaussian spectrum shape $E_{p}(\omega) \propto$ $e^{-(4 \ln 2)\left(\omega-\omega_{0}\right)^{2} / \delta^{2}}$ with the full-width half-maximum (FWHM) $\delta$. The threshold condition needs to be determined first. Using the condition $\lambda_{0}=\gamma / 2$, we obtain the relation between the intracavity threshold power $P_{\text {th }}$ and the pump bandwidth $\delta$ (Fig. 3). Here we assume that the pump and signal modes

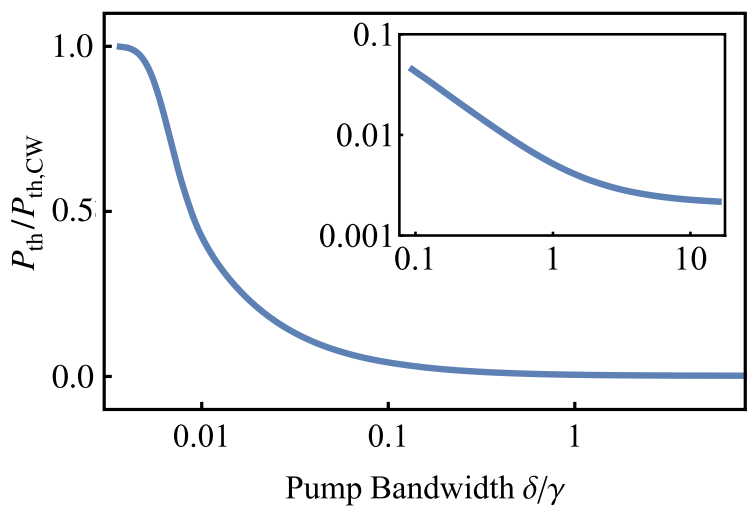

FIG. 3. The intracavity pump threshold power $P_{\text {th }}$ with dependence on the pump bandwidth $\delta$. The power is normalized with $\mathrm{CW}$ intracavity threshold $P_{\mathrm{th}, \mathrm{CW}}=\epsilon \gamma^{2} / 8 \kappa^{2}$, with $\epsilon$ being the absolute permittivity. The inset is the log-scale plot. This figure assumes $\gamma_{p}=2 \gamma$. 

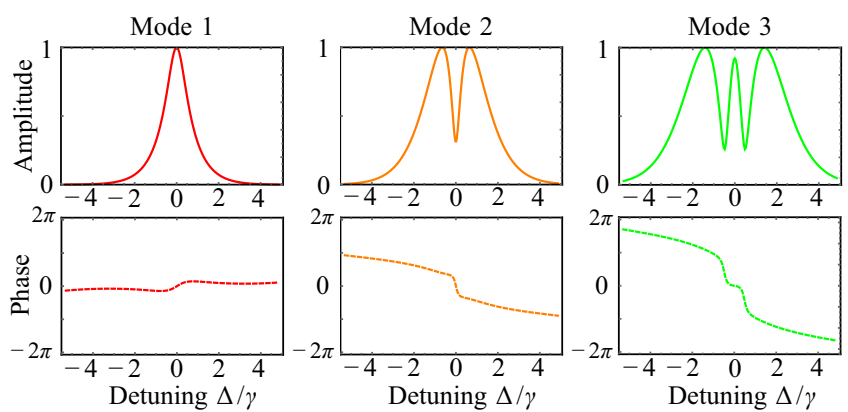

FIG. 4. The spectral amplitude (solid) and phase (dashed) of the first three characteristic modes near pump threshold. This figure assumes $\gamma_{p}=2 \gamma$ and $\delta=2 \gamma_{p}$.

have the same quality factor, thus $\gamma_{p}=2 \gamma$. Monotonic decay of the intracavity threshold power $P_{\text {th }}$ with respect to the pump bandwidth $\delta$ can be observed, due to the contribution from multiple pump-frequency components. Compared with a continuous wave $(\mathrm{CW})$ pump, the intracavity threshold power can be decreased by three orders of magnitude, making this scheme highly power efficient.

Through the Bloch-Messiah decomposition of Eq. (7), we can obtain the spectral shape (Fig. 4) and squeezing amplitude (Fig. 5) of each characteristic mode. Then the squeezing level can be estimated with Eq. (11). The squeezing level of the
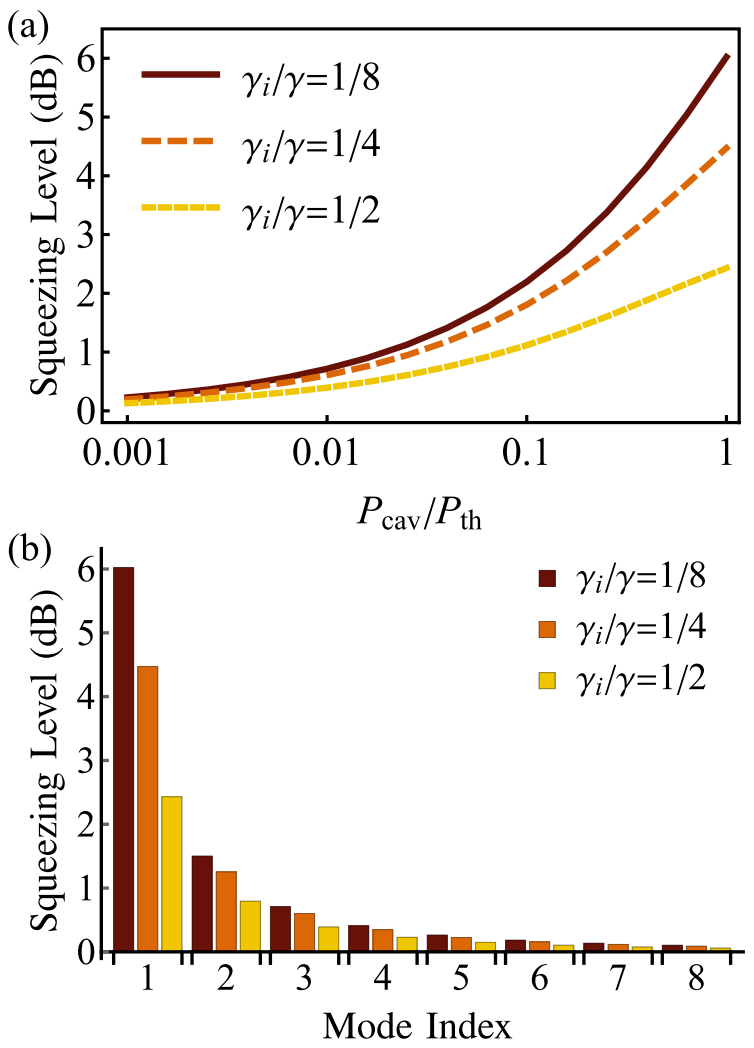

FIG. 5. (a) Squeezing levels of the first characteristic mode with different intracavity power $P_{\text {cav }}$ and intrinsic loss $\gamma_{i}$. (b) Squeezing levels of high-order characteristic modes with intracavity pump power at $P_{\text {cav }}=0.99 P_{\text {th }}$. This figure assumes $\gamma_{p}=2 \gamma$ and $\delta=2 \gamma_{p}$.
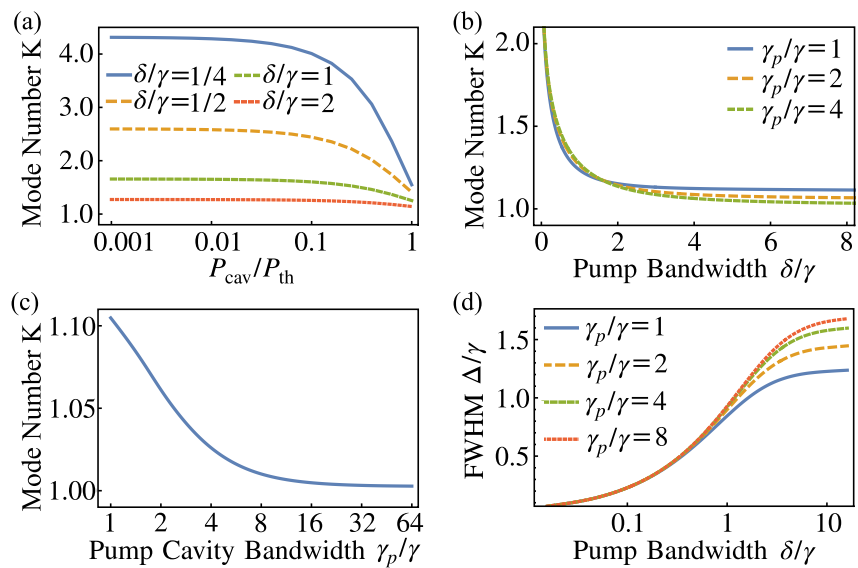

FIG. 6. (a) Effective mode number $K$ as a function of intracavity pump power $P_{\text {cav }} / P_{\text {th }}$ with different pump bandwidth $\delta / \gamma$. This figure assumes $\gamma_{p}=2 \gamma$. (b) Effective mode number $K$ as a function of pump bandwidth $\delta / \gamma$ with different pump cavity bandwidth $\gamma_{p}$. This figure assumes $P_{\text {cav }}=0.99 P_{\text {th }}$. (c) Effective mode number $K$ as a function of pump cavity bandwidth $\gamma_{p}$. This figure assumes $P_{\text {cav }}=0.99 P_{\text {th }}$ and $\delta=16 \gamma$. (d) FWHM of the first characteristic mode $\Delta$ as a function of pump bandwidth $\delta$ with different pump cavity linewidth $\gamma_{p}$. This figure assumes $P_{\text {cav }}=0.99 P_{\text {th }}$.

first characteristic mode is plotted in Fig. 5(a). As expected, the squeezing level increases with pump power below the threshold, and lower intrinsic loss leads to higher squeezing. We further plot the squeezing levels for high-order modes. Because of smaller optical gain, the squeezing levels for highorder modes decrease rapidly [Fig. 5(b)]. Based on Eq. (12), this indicates the output field will have a small effective mode number as well as a high purity without any filtering and postselection. When pump power is small, the effective mode number stays constantly where entangled photon pairs are generated. With the pump power approaching threshold, the effective mode number drops to a value limited by the pump bandwidth [Fig. 6(a)].

We further observe that the effective mode number decreases monotonically with both the input pump bandwidth $\delta$ and the pump cavity linewidth $\gamma_{p}$ [Figs. 6(b) and 6(c)]. The signal cavity with a small linewidth $\gamma$ will function as a spectral-temporal filter to enhance the first characteristic mode and suppress high-order characteristic modes. With a larger input pump bandwidth $\delta$ and pump cavity linewidth $\gamma_{p}$, the filtering effect is more significant, thus leading to a smaller effective mode number. This filtering effect is different from adding narrow filters after the squeezing generation, as the parametric down-conversion and the filtering happen simultaneously in the same cavity. Therefore, no extra loss will be introduced. This filtering effect can be clearly observed in Fig. 6(d), where the FWHM of the first characteristic mode increases rapidly with small pump bandwidths but saturates at large pump bandwidths.

In order to access the maximum squeezing, the spectraltemporal shape of the local oscillator must match the first characteristic mode. Based on the fact that the first characteristic mode shape is critically dependent on the filtering effect 


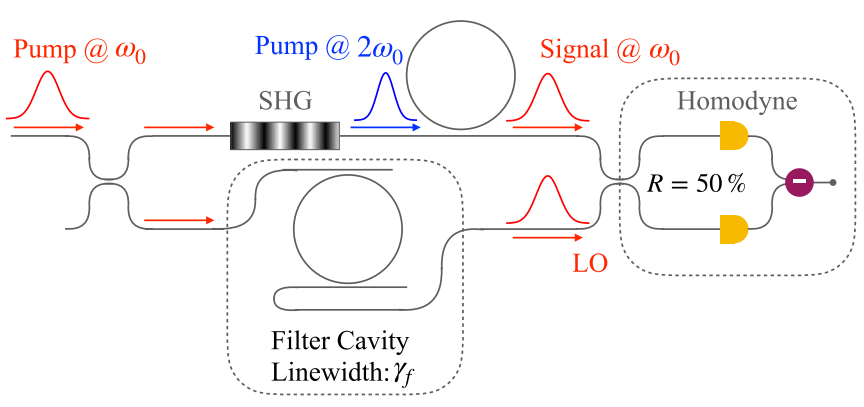

FIG. 7. Proposed setup for local oscillator shaping.

of the signal cavity [Fig. 6(d)], we further design an easy and efficient method for the local oscillator shaping.

As shown in Fig. 7, the generation of the pulsed squeezing follows the standard configuration, where a strong optical pulse at the signal frequency is used to generate the pump pulse for parametric down-conversion. A small portion of the optical pulse is tapped to serve as the local oscillator. In order to match the spectral-temporal shape of the first characteristic mode, the optical pulse simply goes through an optical cavity with Lorentzian line shape. The cavity linewidth $\gamma_{f}$ is optimized to obtain the maximum mode overlap and squeezing level (Fig. 8). With a small pump bandwidth, the system is in the quasi-CW regime, and a local oscillator without any mode shaping can achieve near-perfect mode matching. With a large pump bandwidth, the filtering effect of the signal cavity is significant. A proper filter cavity for the local oscillator is required, and near-perfect matching can be achieved. As a small effective mode number is obtained only with a large pump bandwidth, this approach for the local oscillator shaping should be sufficient.

\section{DISCUSSION}

While the current analysis is based on degenerate parametric down-conversion, the generalization to nondegenerate cases is straightforward. The dynamics of intracavity modes for signal $\hat{a}_{1}$ and idler $\hat{a}_{2}$ can be written as

$$
\begin{aligned}
0= & \int d \omega^{\prime}\left[i\left(\omega-\omega_{k}\right)-\frac{\gamma}{2}\right] \delta\left(\omega-\omega^{\prime}\right) \hat{a}_{k}\left(\omega^{\prime}\right) \\
& +\int d \omega^{\prime} \kappa \varepsilon\left(\omega+\omega^{\prime}\right) \hat{a}_{l}^{\dagger}\left(\omega^{\prime}\right) \\
& +\sqrt{\gamma_{i}} \hat{a}_{k}^{(i)}(\omega)+\sqrt{\gamma_{c}} \hat{a}_{k, i n}(\omega)
\end{aligned}
$$

with the index $(k, l)=(1,2)$ or $(2,1)$. The Bloch-Messiah decomposition needs to be applied to the signal and idler modes simultaneously. All conclusions for degenerate cases remain valid for nondegenerate cases. The pulsed squeezing generation with nondegenerate configuration can be realized with both parametric down-conversion [36] and four-wavemixing [37]. For parametric down-conversion, it is easier to achieve a pump cavity linewidth much larger than its signal cavity linewidth $\left(\gamma_{p} \gg \gamma\right)$, due to the vastly different wavelengths. This is beneficial to achieve a smaller effective mode number. Recent development of aluminum nitride [36,38-40], gallium arsenide [41], and lithium niobate [42-44] photonics has made it possible to demonstrate pulsed squeezing with the
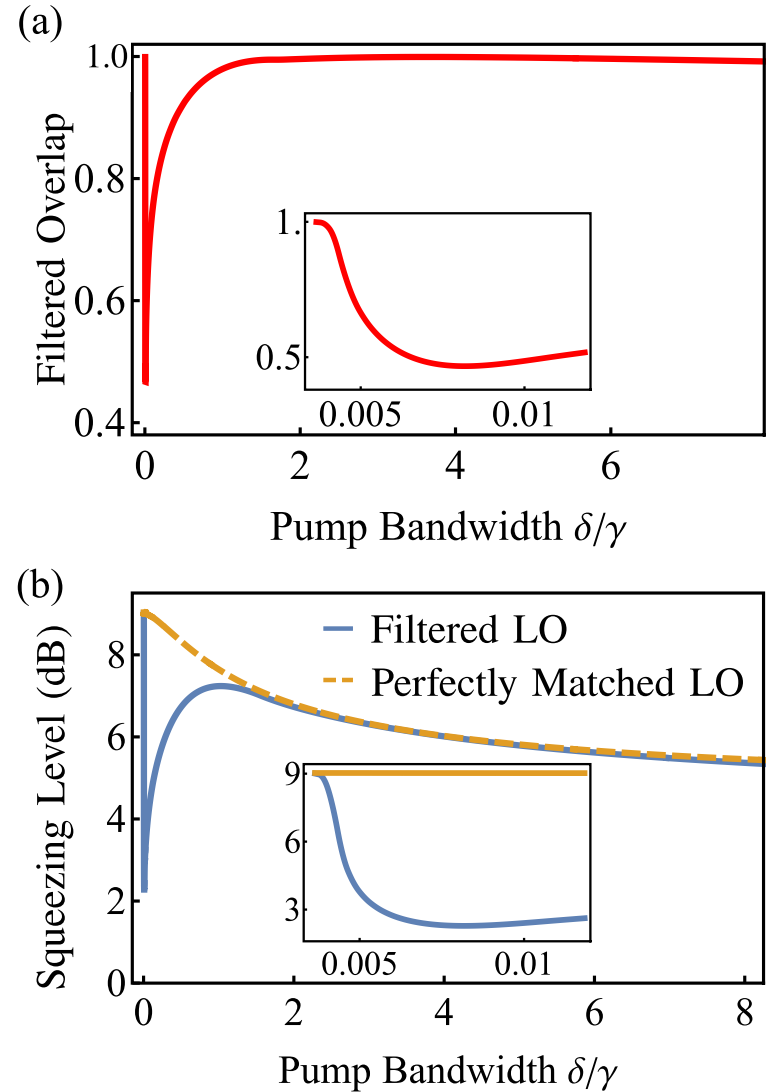

FIG. 8. (a) The overlap between first characteristic mode and the filtered local oscillator with dependence on $\delta$. (b) Measured squeezing level as a function of $\delta$ with filtered (solid blue line) and perfectly matched local oscillator (dashed orange line). Insets are enlargements of the intermediate region between quasi-CW pump and pulsed pump. This figure assumes $P_{\text {cav }}=0.99 P_{\mathrm{th}}, \gamma_{p}=2 \gamma$, and $\gamma_{i}=1 / 8 \gamma$.

proposed method [45]. On the other hand, four-wave mixing has a wider collection of materials as it does not require noncentrosymmetric crystal structures. For four-wave mixing, the pump field in Eq. (3) needs to be modified as

$$
\varepsilon(\omega)=\int d \omega^{\prime} \frac{\sqrt{\gamma_{p c}} E_{p}\left(\omega-\omega^{\prime}\right)}{-i\left(\omega-\omega^{\prime}-\omega_{0}\right)+\frac{\gamma_{p}}{2}} \frac{\sqrt{\gamma_{p c}} E_{p}\left(\omega^{\prime}\right)}{-i\left(\omega^{\prime}-\omega_{0}\right)+\frac{\gamma_{p}}{2}} .
$$

Based on Hydex silica glass, single photons have been generated from nondegenerate four-wave mixing with pulsed pumps [46]. This corresponds to the case that pump power is far below the threshold [Fig. 6(a)]. The experimental result matches our theoretical calculation well [47]. Recently, the $\mathrm{CW}$-squeezed light has also been achieved with photonic ring cavities $[48,49]$, which brightens the way toward integrated pulsed squeezing generation.

\section{CONCLUSION}

In conclusion, we have proposed an approach to generating pulsed squeezing with high temporal purity. The parametric down-conversion in photonic cavities with a single pulsed pump is analyzed based on the Bloch-Messiah decomposition. 
We show that near-unity effective mode number can be obtained. A larger pump cavity linewidth as well as a larger pump bandwidth are preferred to decrease the effective mode number. As the dependence of the effective mode number on pump cavity linewidth and pump bandwidth is monotonic, no delicate balance between the pump power and cavity linewidth is required, making the approach robust. An additional benefit is the low pump threshold due to the contribution from multiple frequency components, leading to the high power efficiency of this approach. We further design an easy method to realize optimum matching between the local oscillator and output characteristic mode for maximum squeezing measurement. The robustness, high efficiency, and easy matching of the local oscillator make this approach promising for large-scale quantum network and complex quantum state generation.

\section{ACKNOWLEDGMENTS}

This work was supported by Office of Naval Research (No. N00014-19-1-2190), National Science Foundation (No. ECCS-1842559 and No. CCF-1907918), and U.S. Department of Energy UT-Battelle/Oak Ridge National Laboratory (No. 4000178321)
[1] C. Weedbrook, S. Pirandola, R. García-Patrón, N. J. Cerf, T. C. Ralph, J. H. Shapiro, and S. Lloyd, Gaussian quantum information, Rev. Mod. Phys. 84, 621 (2012).

[2] T. Opatrný, G. Kurizki, and D.-G. Welsch, Improvement on teleportation of continuous variables by photon subtraction via conditional measurement, Phys. Rev. A 61, 032302 (2000).

[3] A. Ourjoumtsev, A. Dantan, R. Tualle-Brouri, and P. Grangier, Increasing Entanglement Between Gaussian States by Coherent Photon Subtraction, Phys. Rev. Lett. 98, 030502 (2007).

[4] K. Wakui, H. Takahashi, A. Furusawa, and M. Sasaki, Photon subtracted squeezed states generated with periodically poled $\mathrm{KTiOPO}_{4}$, Opt. Express 15, 3568 (2007).

[5] N. Namekata, Y. Takahashi, G. Fujii, D. Fukuda, S. Kurimura, and S. Inoue, Non-Gaussian operation based on photon subtraction using a photon-number-resolving detector at a telecommunications wavelength, Nat. Photon. 4, 655 (2010).

[6] M. Walschaers, C. Fabre, V. Parigi, and N. Treps, Entanglement and Wigner Function Negativity of Multimode Non-Gaussian States, Phys. Rev. Lett. 119, 183601 (2017).

[7] T. C. Ralph, A. Gilchrist, G. J. Milburn, W. J. Munro, and S. Glancy, Quantum computation with optical coherent states, Phys. Rev. A 68, 042319 (2003).

[8] A. Ourjoumtsev, R. Tualle-Brouri, J. Laurat, and P. Grangier, Generating optical Schrödinger kittens for quantum information processing, Science 312, 83 (2006).

[9] J. S. Neergaard-Nielsen, B. M. Nielsen, C. Hettich, K. Mølmer, and E. S. Polzik, Generation of a Superposition of Odd Photon Number States for Quantum Information Networks, Phys. Rev. Lett. 97, 083604 (2006).

[10] W. Asavanant, K. Nakashima, Y. Shiozawa, J.-I. Yoshikawa, and A. Furusawa, Generation of highly pure Schrödinger's cat states and real-time quadrature measurements via optical filtering, Opt. Express 25, 32227 (2017).

[11] D. Gottesman, A. Kitaev, and J. Preskill, Encoding a qubit in an oscillator, Phys. Rev. A 64, 012310 (2001).

[12] N. C. Menicucci, P. van Loock, M. Gu, C. Weedbrook, T. C. Ralph, and M. A. Nielsen, Universal Quantum Computation with Continuous-Variable Cluster States, Phys. Rev. Lett. 97, 110501 (2006).

[13] N. C. Menicucci, Fault-Tolerant Measurement-Based Quantum Computing With Continuous-Variable Cluster States, Phys. Rev. Lett. 112, 120504 (2014).

[14] W. Asavanant, Y. Shiozawa, S. Yokoyama, B. Charoensombutamon, H. Emura, R. N. Alexander, S.
Takeda, J.-i. Yoshikawa, N. C. Menicucci, H. Yonezawa et al., Generation of time-domain-multiplexed two-dimensional cluster state, Science 366, 373 (2019).

[15] B. Q. Baragiola, G. Pantaleoni, R. N. Alexander, A. Karanjai, and N. C. Menicucci, All-Gaussian Universality and Fault Tolerance With the Gottesman-Kitaev-Preskill Code, Phys. Rev. Lett. 123, 200502 (2019).

[16] O. Pfister, Continuous-variable quantum computing in the quantum optical frequency comb, J. Phys. B 53, 012001 (2019).

[17] C. Cui, K. P. Seshadreesan, S. Guha, and L. Fan, HighDimensional Frequency-Encoded Quantum Information Processing With Passive Photonics and Time-Resolving Detection, Phys. Rev. Lett. 124, 190502 (2020).

[18] C. N. Gagatsos and S. Guha, Efficient representation of Gaussian states for multimode non-Gaussian quantum state engineering via subtraction of arbitrary number of photons, Phys. Rev. A 99, 053816 (2019).

[19] D. Su, C. R. Myers, and K. K. Sabapathy, Conversion of Gaussian states to non-Gaussian states using photon-numberresolving detectors, Phys. Rev. A 100, 052301 (2019).

[20] G. Triginer Garces, H. M. Chrzanowski, S. Daryanoosh, V. Thiel, A. L. Marchant, R. B. Patel, P. C. Humphreys, A. Datta, and I. A. Walmsley, Quantum-enhanced stimulated emission detection for label-free microscopy, Appl. Phys. Lett. 117, 024002 (2020).

[21] R. E. Slusher, P. Grangier, A. LaPorta, B. Yurke, and M. J. Potasek, Pulsed Squeezed Light, Phys. Rev. Lett. 59, 2566 (1987).

[22] C. Kim and P. Kumar, Quadrature-Squeezed Light Detection Using a Self-Generated Matched Local Oscillator, Phys. Rev. Lett. 73, 1605 (1994).

[23] Y. Eto, T. Tajima, Y. Zhang, and T. Hirano, Observation of squeezed light at $1.535 \mu \mathrm{m}$ using a pulsed homodyne detector, Opt. Lett 32, 1698 (2007).

[24] A. Eckstein, A. Christ, P. J. Mosley, and C. Silberhorn, Highly Efficient Single-Pass Source of Pulsed Single-Mode Twin Beams of Light, Phys. Rev. Lett. 106, 013603 (2011).

[25] U. L. Andersen, T. Gehring, C. Marquardt, and G. Leuchs, Thirty years of squeezed light generation, Phys. Scr. 91, 053001 (2016).

[26] W. Wasilewski, A. I. Lvovsky, K. Banaszek, and C. Radzewicz, Pulsed squeezed light: Simultaneous squeezing of multiple modes, Phys. Rev. A 73, 063819 (2006). 
[27] G. Patera, N. Treps, C. Fabre, and G. J. De Valcarcel, Quantum theory of synchronously pumped type i optical parametric oscillators: Characterization of the squeezed supermodes, Eur. Phys. J. D 56, 123 (2010).

[28] S. Jiang, N. Treps, and C. Fabre, A time/frequency quantum analysis of the light generated by synchronously pumped optical parametric oscillators, New J. Phys. 14, 043006 (2012).

[29] Y. Eto, T. Tajima, Y. Zhang, and T. Hirano, Observation of quadrature squeezing in a $\chi$ (2) nonlinear waveguide using a temporally shaped local oscillator pulse, Opt. Express 16, 10650 (2008).

[30] C. Bloch and A. Messiah, The canonical form of an antisymmetric tensor and its application to the theory of superconductivity, Nucl. Phys. 39, 95 (1962).

[31] S. L. Braunstein, Squeezing as an irreducible resource, Phys. Rev. A 71, 055801 (2005).

[32] D. F. Walls and G. J. Milburn, Quantum Optics (Springer Science \& Business Media, Berlin, 2007).

[33] M. Aspelmeyer, T. J. Kippenberg, and F. Marquardt, Cavity optomechanics, Rev. Mod. Phys. 86, 1391 (2014).

[34] P. D. Drummond and Z. Ficek, Quantum Squeezing, Springer Series on Atomic, Optical, and Plasma Physics (Springer Science \& Business Media, Berlin, 2013), Vol. 27.

[35] A. Christ, K. Laiho, A. Eckstein, K. N. Cassemiro, and C. Silberhorn, Probing multimode squeezing with correlation functions, New J. Phys. 13, 033027 (2011).

[36] X. Guo, C.-1. Zou, C. Schuck, H. Jung, R. Cheng, and H. X. Tang, Parametric down-conversion photon-pair source on a nanophotonic chip, Light Sci. Appl. 6, e16249 (2017).

[37] M. Kues, C. Reimer, J. M. Lukens, W. J. Munro, A. M. Weiner, D. J. Moss, and R. Morandotti, Quantum optical microcombs, Nat. Photon. 13, 170 (2019).

[38] C. Xiong, W. H. P. Pernice, X. Sun, C. Schuck, K. Y. Fong, and H. X. Tang, Aluminum nitride as a new material for chip-scale optomechanics and nonlinear optics, New J. Phys. 14, 095014 (2012).

[39] L. Fan, C.-L. Zou, M. Poot, R. Cheng, X. Guo, X. Han, and H. X. Tang, Integrated optomechanical single-photon frequency shifter, Nat. Photon. 10, 766 (2016).

[40] L. Fan, C.-L. Zou, R. Cheng, X. Guo, X. Han, Z. Gong, S. Wang, and H. X. Tang, Superconducting cavity electrooptics: A platform for coherent photon conversion between superconducting and photonic circuits, Sci. Adv 4, eaar4994 (2018).
[41] B. Guha, F. Marsault, F. Cadiz, L. Morgenroth, V. Ulin, V. Berkovitz, A. Lemaître, C. Gomez, A. Amo, S. Combrié et al., Surface-enhanced gallium arsenide photonic resonator with quality factor of $6 \times 10^{6}$, Optica 4, 218 (2017).

[42] R. Luo, H. Jiang, S. Rogers, H. Liang, Y. He, and Q. Lin, On-chip second-harmonic generation and broadband parametric down-conversion in a lithium niobate microresonator, Opt. Express 25, 24531 (2017).

[43] C. Wang, C. Langrock, A. Marandi, M. Jankowski, M. Zhang, B. Desiatov, M. M. Fejer, and M. Lončar, Ultrahigh-efficiency wavelength conversion in nanophotonic periodically poled lithium niobate waveguides, Optica 5, 1438 (2018).

[44] J. Lu, J. B. Surya, X. Liu, A. W. Bruch, Z. Gong, Y. Xu, and H. X. Tang, Periodically poled thin-film lithium niobate microring resonators with a second-harmonic generation efficiency of 250,000\%/w, Optica 6, 1455 (2019).

[45] Degenerate parametric down-conversion has been realized based on AIN ring cavities [50]. The linewidths for the signal and pump resonances are 192 and $960 \mathrm{MHz}$ respectively. If pumped by Gaussian pulses with bandwidth $\delta=1 \mathrm{GHz}$, our model predicts that the output mode could have an effective mode number $K=1.038$.

[46] M. Kues, C. Reimer, P. Roztocki, L. R. Cortés, S. Sciara, B. Wetzel, Y. Zhang, A. Cino, S. T. Chu, B. E. Little et al., On-chip generation of high-dimensional entangled quantum states and their coherent control, Nature (London) 546, 622 (2017).

[47] In Ref. [46], Hydexsilica cavity with $\gamma_{p}=\gamma=0.803 \mathrm{GHz}$ is pumped with pulse (duration $0.6 \mathrm{~ns}$ and bandwidth $1.67 \mathrm{GHz}$ ). The effective mode number is measured to be 1.086 . Based on our theoretical mode, the effective mode number of $K=1.092$ is calculated in the low-power regime, which matches the experiment.

[48] Y. Zhao, Y. Okawachi, J. K. Jang, X. Ji, M. Lipson, and A. L. Gaeta, Near-Degenerate Quadrature-Squeezed Vacuum Generation on a Silicon-Nitride Chip, Phys. Rev. Lett. 124, 193601 (2020).

[49] V. D. Vaidya, B. Morrison, L. G. Helt, R. Shahrokshahi, D. H. Mahler, M. J. Collins, K. Tan, J. Lavoie, A. Repingon, M. Menotti et al., Broadband quadrature-squeezed vacuum and nonclassical photon number correlations from a nanophotonic device, Sci. Adv. 6, eaba9186 (2020).

[50] A. W. Bruch, X. Liu, J. B. Surya, C.-L. Zou, and H. X. Tang, On-chip $\chi^{(2)}$ microring optical parametric oscillator, Optica 6 , 1361 (2019). 